\title{
Nanocrystal optoelectronics for quality lighting and displays (ID: 1780136)
}

\author{
Swee Tiam Tan ${ }^{1}$ and Hilmi Volkan Demir ${ }^{1,2}$
}

${ }^{1}$ LUMINOUS! Centre of Excellence for Semiconductor Lighting and Displays, School of Electrical and Electronic Engineering, and School of Physical and Mathematical Sciences, Nanyang Technological University - NTU Singapore

${ }^{2}$ Electrical and Electronics Engineering, Physics, UNAM-Institute of Materials Science and Nanotechnology, Bilkent University, Ankara, Turkey 06800, Turkey

\section{$\underline{\text { Abstract }}$}

We review the recent advances in color-conversion LEDs integrating nanophosphors of semiconductor quantum dots for quality lighting and displays in our group.

To combat environmental issues, combined with the energy problem of limited resources, innovating fundamentally new ways of raising energy efficiency is essential to our energy future. Today achieving lighting efficiency is an important key because artificial lighting consumes about $19 \%$ of global energy generation. There is a large room for improving energy utilization of lighting. However, the scientific challenge is to reach simultaneously highquality photometric performance [1]. To address these and related problems [2], we develop and demonstrate new classes of color-conversion LEDs integrating nanophosphors of semiconductor quantum dots for high-photometric quality [3] and those enhanced using excitonics (controlling exciton-exciton interactions) through Förster-type nonradiative energy transfer (NRET) [4]. We study intrinsic performance limits and fundamental photometric tradeoffs of such narrow-emitter nanophosphors investigating a large scale $(>200 \mathrm{M})$ of designs [5]. We show that it is possible to achieve high levels of photometric performance with a luminous efficacy of optical radiation (LER) $>380 \mathrm{~lm} / \mathrm{W}_{\text {opt }}$ and a color-rendering index (CRI) >90 in the warm-white region, important for spectrally high-efficiency, high-quality lighting [6]. We also show eco-friendly nanocrystals for color-conversion LEDs [7] and largearea sheets of such nanocrystals for remote phosphor applications [8].

The eye has always been known responsible for our visual perception (see Figure 1). Today, the mechanisms and physiological pathways of the vision process have been well understood. There are two photoreceptors located in the human eye that are responsible for visual perception. The color perception basically emanates from the activity of the red, green, and blue sensitive cones, which are active at higher luminance levels (photopic vision regime). On the other hand, while rods do not contribute to the color perception, their effect on visual perception is dominant only under dim light (scotopic vision regime). When the illumination level is between these two regimes, (i.e., mesopic vision conditions), these two photoreceptors work together for delivering the visual information to the brain. The corresponding eye sensitivity functions for photopic, mesopic (at a luminance of $0.5 \mathrm{~cd} / \mathrm{m}^{2}$ ), and scotopic vision regimes are shown in Figure 2. The problems of low scotopic/photopic (S/P) ratio lighting and low CRI can be solved by modifying the phosphors. This requires design and synthesis of new phosphors, for example, by using nanophosphors of colloidal semiconductor quantum dots (QDs). Due to the size tenability and narrow emission bandwidth ( $\sim 30 \mathrm{~nm})$, QD luminophors in white LEDs enable precise spectrum engineering to realize high-quality lighting tailored for different lighting requirements. Moreover, colloidal QDs feature large photoluminescence quantum yield in solution (reasonable yield in film) and increasing absorption below its emission peak toward shorter wavelengths. 


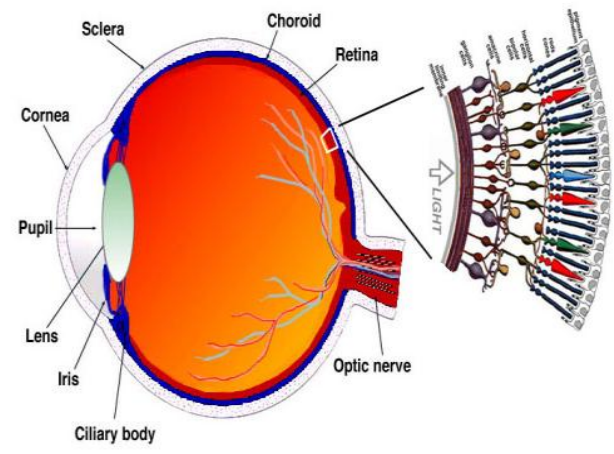

Figure 1. A cross-section schematic of a human eye with a magnified view of the retina [9].

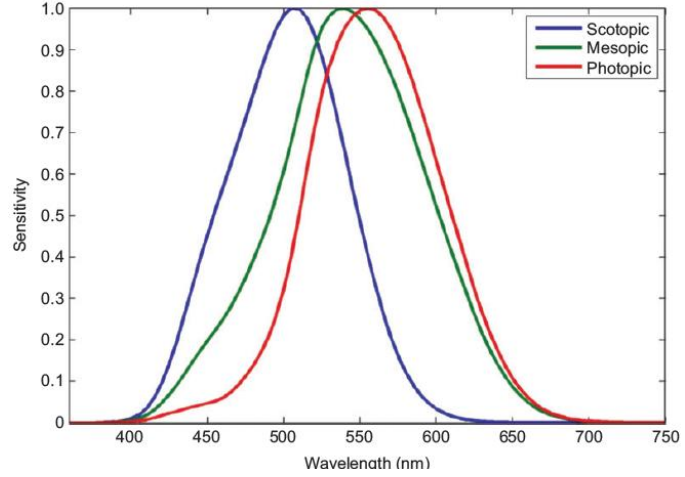

Figure 2. Eye sensitivity function in different vision regimes: photopic (red), mesopic (green, at a luminance of $0.5 \mathrm{~cd} / \mathrm{m}^{2}$ ), and scotopic (blue) eve sensitivitv functions 「101.

In fact, tremendous effort has been devoted into QD-white LEDs following the first demonstrations of the use of color-converting QDs on LEDs [11-14]. In our studies, we have found out that using QDs one can theoretically achieve a white LED having CRI >90, LER $>380 \mathrm{~lm} / \mathrm{W}_{\text {opt }}$ and CCT $<4000 \mathrm{~K}$ [15]. A recommended white light spectrum and its performance are given in Figure 3. The outcome of this particular work shows that optimization of all of the important photometric properties of a white light source can be realized using these novel nanophosphors of quantum dots as opposed to conventional phosphors. Moreover, we found out the relations of the photometric performance of a quantum dot integrated white LED which is very critical for the design of the emission spectrum [15]. Based on these theoretical studies, we developed a white LED using CdSe/ZnS core/shell quantum dots having an extraordinary photometric performance: CRI=89.2, $\mathrm{CCT}=2982 \mathrm{~K}$ and LER=357 lm/ $\mathrm{W}_{\text {opt }}$ [6]. Corresponding emission spectrum of the white LED can be found in Figure 4. This is the state-of-the art white LED in terms of photometric performance. Furthermore, in this work we demonstrated the tunability of photometric performance the quantum dot integrated LEDs. By controlling the quantum dot amounts in the color conversion layer, we managed to preserve high CRI and LER by obtaining warmer shades of white light emission whose CCT values drop down to $2390 \mathrm{~K}$.

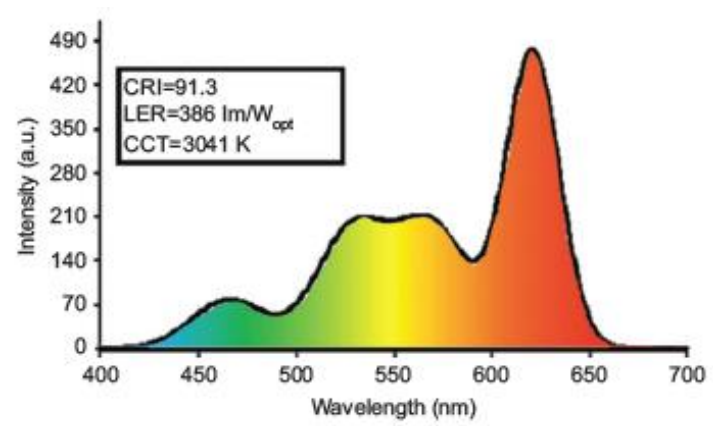

Figure 3. QD-WLED spectrum generated using the average values obtained by applying thresholds of CRI $>90$, LER $>380$ $1 \mathrm{~m} / \mathrm{W}_{\text {opt }}$, and CCT $<4000 \mathrm{~K}$ along with its photometric performance [15].

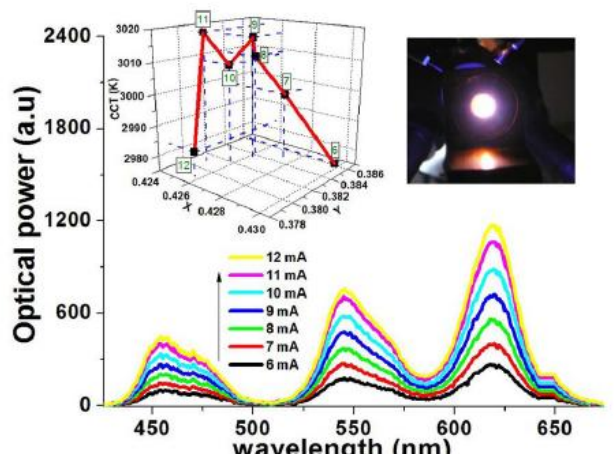

Figure 4. Experimental demonstration of a quantum dot integrate white LED using $\mathrm{CdSe} / \mathrm{ZnS}$ quantum dots. This LED has a CRI of 89.2, a CCT of $2982 \mathrm{~K}$ and a LER of $357 \mathrm{~lm} / \mathrm{W}_{\text {opt }}[6]$. 
Environmental friendly QD emitters have also been accomplished in our group. We synthesized high-quality InP/ZnS core-shell QDs with luminescence tunable over the entire visible spectrum [7]. Separately, we also demonstrated a very large-area (over $50 \mathrm{~cm} \times 50 \mathrm{~cm}$ ) freestanding optical membranes of colloidal InP/ZnS QDs [8]. The freestanding QD film was further utilized as a remote color-converting nanophosphor sheet together with a blue InGaN/GaN LED chip, demonstrating high photometric quality white LED (see Figure 5).
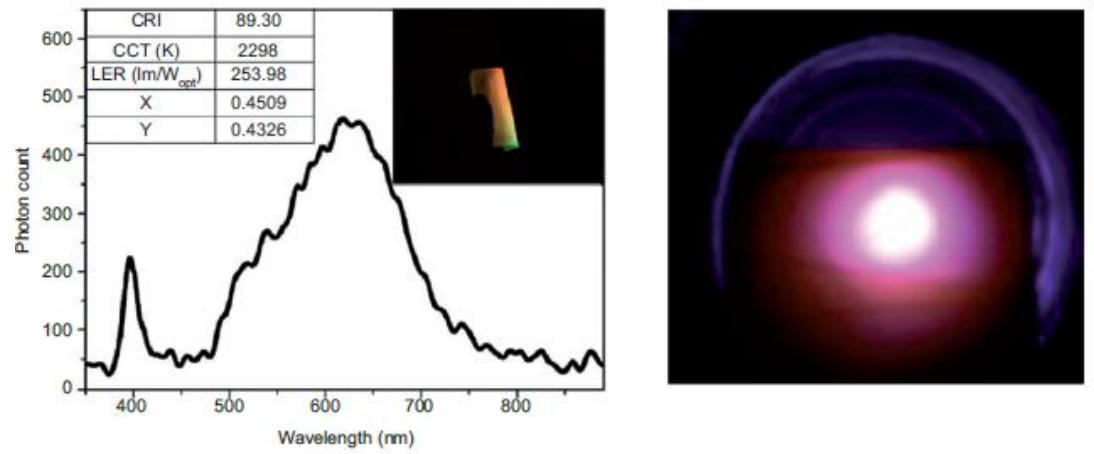

Figure 5. Electroluminescence spectra of a white LED using an InP/ZnS QD sheet as the remote color-converting nanophosphors together with a blue InGaN/GaN LED chip [8].

In conclusion, we have demonstrated a feasible solution addressing the issue of simultaneously achieving high energy efficiency and high-quality photometric performance lighting through InGaN/GaN blue LED chip integrated with colloidal semiconductor QD nanoluminophors. This approach is expected to benefit the design of different emitters for various applications, for example, indoor lighting, outdoor lighting, and display emitters.

Acknowledgement:

This work is supported by the National Research Foundation of Singapore under Grant No. NRF-CRP-6-2010-2 and NRF-RF-2009-09 and the Singapore Agency for Science, Technology and Research (A*STAR) SERC under Grant No. 1121202009.

\section{References:}

[1] H. V. Demir, S. Nizamoglu, T. Erdem, E. Mutlugun, N. Gaponik, and A. Eychmuller, Nano Today 6, $632(2011)$.

[2] T. Erdem, and H. V. Demir, Nature Photonics 5, 126 (2011).

[3] S. Nizamoglu, G. Zengin, and H. V. Demir, Applied Physics Letters 92, 031102 (2008).

[4] S. Nizamoglu and H.V. Demir, Applied Physics Letters 95, 151111 (2009).

[5] T. Erdem, S. Nizamoglu, and H. V. Demir, Optics Express 20, 3275 (2012).

[6] S. Nizamoglu, T. Erdem, X. W. Sun, and H. V. Demir, Optics Letters 35, 20, 3372-3374 (2010).

[7] X. Y. Yang, D. W. Zhao, K. S. Leck, F. Lu, S. T. Tan, Y. X. Tang, J. L. Zhao, H. V. Demir, and X.

W. Sun, Advanced Materials 24, 4180 (2012).

[8] E. Mutlugun, P. L. Hernandez-Martinez, C. Eroglu, Y. Coskun, T. Erdem, V. K. Sharma, E. Unal, S.

K. Panda, S. G. Hickey, N. Gaponik, A. Eychmuller, and H. V. Demir, Nano Letters 12, 3986 (2012).

[9] Webvision, The Organization of the Retina and Visual System, http://webvision.med.utah.edu/book/part-i-foundations/simple-anatomy-of-the-retina/

[10] T. Erdem and H.V. Demir, Nanophotonics 2, 57 (2013).

[11] M. Achermann, M. A. Petruska, D. D. Koleske, M. H. Crawford, and V. I. Klimov, Nano Letters 6, 1396 (2006).

[12] H. S. Chen, C. K. Hsu, and H. Y. Hong, IEEE Photonics Technology Letters 18, 193 (2006).

[13] H. S. Chen, D. M. Yeh, C. F. Lu, C. F. Huang, W. Y. Shiao, J. J. Huang, C. C. Yang, I. S. Liu, and W. F. Su, IEEE Photonics Technology Letters 18, 1430 (2006).

[14] S. Nizamoglu, T. Ozel, E. Sari, and H. V. Demir, Nanotechnology 18, 065709 (2007).

[15] T. Erdem, S. Nizamoglu, X. W. Sun, and H. V. Demir, Optics Express, 18, 340 (2010). 